\title{
Activation of the primary visual cortex (V1) related to surface brightness
}

\author{
Hidehiko Komatsu \\ National Institute for Physiological Sciences and the Graduate University for Advanced Studies \\ (SOKENDAl), Myodaiji, Okazaki, Aichi, Japan. e-mail: komatsu@nips.ac.jp
}

Cornelissen and Vladusich claim that recent fMRI studies ${ }^{1,2}$ did not provide evidence that activity in the early visual cortex corresponds in a topographic (isomorphic) manner with illusory surface regions. I first point out that, in my review ${ }^{3}$, I have argued that neuron activities in the primary visual cortex (V1) during filling-in at the blind spot ${ }^{4}$ is not consistent with the isomorphic theory and suggested a scale-sensitive mechanism as an alternative possibility. I think that the presence of activation in the region representing the surface interior should be distinguished from the question of whether the mechanism of filling-in is isomorphic or not.

Apart from this general point, it remains a puzzle as to why these authors did not find that contrastdependent activation in V1 was related to surface brightness ${ }^{1}$, even though the activities of some neurons in the monkey and cat V1 with receptive fields inside the surface are modulated by the luminance change in the surround in a way that is consistent with the brightness induction ${ }^{5,6}$. There are two possible explanations. One possibility is related to the assumption of their model. These authors assumed that signals related to filling-in are constant across the surface interior, but this seems to be an unnatural assumption. Signals related to filling-in are generated at the edge of the surface, and they should decay from the edge towards the centre of the surface. Another possibility is that, in their fMRI measurements, the activities of neurons with different properties could be cancelled by each other. It has been shown that some V1 neurons are excited by bright surfaces and inhibited by dark surfaces (photergic ${ }^{7}$ or bright-type neurons ${ }^{6}$ ). Some others are excited by dark surfaces and inhibited by bright surfaces (scotergic or darktype neurons). If the luminance of a large surface is temporally modulated sinusoidally, then the activities of these two types of neuron, which have receptive fields inside the surface, are modulated anti-phase: when the luminance is high, bright-type neurons are activated whereas dark-type neurons are suppressed; however, when the luminance is low, the opposite is true. Similarly, two types of neuron exist that are either activated or suppressed by the change in the surround luminance ${ }^{6}$. The activities of these two types of neuron could be cancelled by each other when the summed activities are balanced in the opposite direction (activity increase versus decrease). If this happens, no signal will be detected when the population activities of both types of neuron are measured (as in fMRI measurement) even though activities of individual neurons can be detected by neural recording experiments. The relative number of the two types of cell and nonlinearity in the luminanceresponse relationship, together with other factors, might have arole in determining the total balance of activation.

There is no doubt that neural signals generated by uniform surfaces are much weaker than those generated by contrast borders ${ }^{1,9}$. Signals related to filling-in should be even smaller, despite their significance in our perception. Finding the neural signals related to filling-in and elucidating their precise nature is like finding a tiny jewel among the grains of sand on the beach, and it should remain a challenge in visual neuroscience.

1. Cornelissen, F. W., Wade, A. R., Vladusich, T., Dougherty, R. F. \& Wandell, B. A. No functional magnetic resonance imaging evidence for brightness and color filling -in in early human visual cortex. J. Neurosci. 26, $3634 \llbracket 3641$ (2006).

2. Perna, A., Tosetti, M., Montanaro, D. \& Morrone, M. C. Neuronal mechanisms for illusory brightness perception in humans. Neuron 47, 645651 (2005).

3. Komatsu, H. The neural mechanisms of perceptual filling -in. Nature Rev. Neurosci. 7 ,

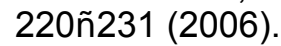

4. Komatsu, H., Kinoshita, M. \& Murakami, I. Neural responses in the retinotopic representation of the blind spot in the macaque $\mathrm{V} 1$ to stimuli for perceptual filling -in. $J$. Neurosci. 20, 93109319 (2000).

5. Rossi, A. F., Rittenhouse, C. D. \& Paradiso, M. A. The representation of brightness in primary visual cortex. Science 273, $1104 \square 1107$ (1996). 
6. Kinoshita, M. \& Komatsu, H. Neural representation of the luminance and brightness of a uniform surface in the macaque primary visual cortex. J. Neurophysiol. 86, $2559 \square 2570$ (2001).

7. Kayama, Y., Riso, R. R., Bartlett, J. R. \& Doty, R. W. Luxotonic responses of units in macaque striate cortex. J. Neurophysiol. 42, $1495 \square 1517$ (1979).

8. Haynes, J. D., Lotto, R. B. \& Rees, G. Responses of human visual cortex to uniform surfaces. Proc. Natl Acad. Sci. USA 101, $4286 \square 291$ (2004).

9. Friedman, H. S., Zhou, H. \& von der Heydt, R. The coding of uniform colour figures in monkey visual cortex. J. Physiol. 548, 593613 (2003). 